\title{
Analysis of Accordion Application in movie Art
}

\author{
Zhenyu $\mathrm{Hu}$ \\ Music School, Nanyang Institute of Technology, Nanyang Henan 473000, China
}

Keywords: Movie art, Accordion play, Background music.

\begin{abstract}
The essence of art is a kind of communication behavior. As the important content and component of the development of movie art, background music integrates the arts including movie language, drama performance, movie special effects together harmoniously. It can be said to be the path for the movie aesthetic system to be run and created orderly and organically. Only by well integrating the music and the whole movie can deep uninterrupted communication between audience and the movie be better guided. This article analyzes the important role movie background music plays in movie art by taking accordion as the entry point.
\end{abstract}

\section{Introduction}

As an important functional aesthetic setting in movie art, movie background music has been developed from pure background music art to a skilled technical performance means in the late phase, till gradually developed into the current one kind of aesthetic elements to measure the movie expression. It has become a necessary part of movie art. Accordion originated in western countries. With the development of economic globalization, the mutual infiltration between Chinese and western culture, accordion music has been quickly spread to all over the world and become a musical instrument popular in each nation and loved by each nation. In the field of movie background music, the accordion playing way is also used by more and more moviemakers.

\section{The use of accordion music highlight the style of movie}

Generally speaking, as for the movie, the movie background music has always been in the position of "background", this also matches its name "movie background music". However, the difference between primary and secondary will change sometimes. Exquisite background music in some movies often can surpass its position of "background" and be promoted to the position of "theme". The change of position depends on the contribution of its function, if the movie background music itself could be integrated in the plot of movie perfectly and even influence the movie's definition of theme and style without affecting the audience's understanding of the movie theme, the judgment of movie style mainly depend on the style of movie background music, the position of movie background music would be promoted to the position of "theme".

In the movie, Amelie from Montmartre which was done by the master of background music--Tiersen himself, as the soul of the movie, the background music played by accordion combined with other music elements such as guitar, piano and violin etc. forms a well arranged and understanding assembler style in the movie. Acoustic music renders the plot. In playing accordion, the basic style of the movie is full of romantic color of French amorous feelings. Concise and pure background music style forms meticulous aesthetic flavor, sending a inner music intangible magic virtually. It goes into the audience's heart subtly triggering viewers' collective resonance. The two classical background music, the Place I never know and the climax of the movie, Submerging, at the beginning of the movie, mainly use accordion playing. Movie content and accordion playing foil each other, forming a kind of emotional tone which is obscure with well foiling and rendering the whole content, creating a kind of special atmosphere with organic integration of characters' genuine feeling and music, ensuring the smoothness of the movie story. The use of the accordion cleverly unfold the characters' emotion, better playing the role of expressing the movie theme. In the movie when Emily met the blind man, the melody of accordion gradually changed from slow to fast according to the 
rhythm of the movie story and then the suddenly stopped and interrogative music form leaves audience enough free imagination space, sublimating the theme of the movie, very much conforming to the romantic color of the movie as well as corresponding to French people' feelings of pursuit of romantic.

The animator in the Netherlands, MichaelDudokdeWit, won the 2001 Oscar awards for best animated short movie by his Father and Daughter. The whole time of the movie is just eight and a half minutes. But it had attracted great attention once opening. Dialogue and comment do not appear in the animation movie. Its background music is played mainly by instrument of an accordion. Simple images mainly tell a simple story after girl leaves her father. The ferry where the girl and her father part is the main place of the story. With the scene description of little girls' back and forth waiting for father to return back in the process of growing, combined with sorrow and sweet music of accordion, what they together reveal is the eternal theme of love and affection.

In Happy Together, because of being gays, He Baorong and Li Yaohui can't integrate themselves into the social communication in HongKong, so they take the guise of tourism as an excuse for leaving the city and go to a city of Argentina. In the strange city of Argentina, two people often have conflicts because of a few small problems due to the different personalities and finally feel more lonely because the finally emotional crisis generated by conflicts. In the use of movie background music, Wong Kar-wai uses the background music of Bandoneon accordion of Astor Pantaleón Piazzolla, a Latin American musician to express the loneliness between group and individual character and reveal the deep contradictions between sociality and loneliness in the special homosexual group. movie Postman reflects the life history of Pablo Neruda, the winner of Nobel Prize in literature, left-wing progressive poet in Chile. The composer, Luis Enrique Bacalov mainly use Bundoran accordion to play the background music. The total effect of the background music of the movie shows a kind of quiet, simple but elegant, beautiful atmosphere to penetrate gentle touch in people's heart. Concise and lovely accordion playing with agrestic breath expresses the natural state of mind, showing the warmth of human at subtleties as well as intricately woven together with the theme of the movie story.

\section{The directive function of accordion music in the movie}

The choice of background music for a movie should coordinate with the requirement of various elements such as the plot, scene and character setting of the movie to play the role of foiling atmosphere and guiding the story direction. The essence of art is a kind of communication behavior, as a communication bridge between the movie and the story, music integrates arts of movie language, drama performance, movie special effects etc. together harmoniously. Therefore, only when music and the whole movie are integrated together well, can deep uninterrupted communication between audience and movie be better guided.

At the opening of movie the Butterfly guided by the leading figure of "post-new-wave" of Taiwanese movie, Tso-chi Chang, heroine's hokkien monologue in the lonely accordion music with simple cooperation has well communicated the distance between the audience and the movie as well as made audience be very easy to go into the heart of the heroine to feel her inner thoughts. At the same time it also guides the direction of the movie story: Hope will fall thick in the blast, all people are in deep desperation, unable to be redeemed. When the movie Wonderful Life with Sun Hai Ying, Lv Liping as the starring roles during the process of expressing the complex inner emotion changes of empty-nest elderly people, it adopts accordion music to change the towards of movie emotion. From the original brick dance with loudspeakers' background music to old Feng playing accordion and his wife's death, the change of background music continuously integrated with the change of master's emotion. This well guides the movie plot and subtle variations of the inner world of characters.

In the movie Amelie from Montmartre, unique lens language makes the viewer well involved in the occurrence of the story, but there are a lot of close visual scenes in the movie, which is easy to make people have "oppressing sensation" brought by "safe distance" and thus affect the audience's viewing 
mood. When attracting the audience to have both visual and auditory enjoyment, the subtle accordion music at the same time greatly alleviates the oppressive feeling the movie brings the audience, the beating notes and melodies guide the viewers to follow the rhythm of the movie in the joyful experience to participate in, to feel, to understand, to think the profound meanings the movie really interprets.

It is just the accordion music which keeps audience feelings always in the theme and the story of movie. This is also the reflection of important function of movie background music. Only the dynamic fit between the form of music and the movie rhythm can correspondences and communications between different rhythms in the movie be perfected to keep the movie's fluency and penetration.

\section{The use of accordion music and shaping the movie's characters}

The background music in a good movie not only can render, foil the theme of the movie, but also should be a better preparation for shaping characters. In the movie work Peacock directed by Gu Changwei, because of its unique narrative art and the exquisite acting of actors winning high praise from all parties, successively winning various awards such as the silver bear award in Berlin movie festival, Best Actress of Shanghai movie critics award, Chinese movie media best director award, best actress award etc., it has received much concern, making the reputation of actress Zhang Jingchu.

The movie Peacock focuses on a story of sister, elder brother and younger brother of an ordinary families in north about dreams, love, and life. Among which the story of sister Gao Wei Hong played by Zhang Jingchu is the main line. Movie show from the start the scene of the heroine sister Gao Weigong's playing accordion. The focus of the scene lies not at how exquisite and delicate sister's playing technique is, but at how she throws herself into the playing. Accordion and music make sister completely immersed in his dream, even there is already a boiling kettle besides her with kettle cover ringing loudly by the steam can't influence the accordion in her hands. The movie interprets the pure persistent personality of sister through the clever show of accordion. Besides, the movie music accordion playing Footpath in the movie not only highlights the movie narrative tone well but also corresponds to the story structure of from sister's having a dream to dream is disillusioned, successfully modeling the hidden strong and brave character of the sister with gentle appearance. The accordion in the movie Peacock is not only the prop and background music of the movie, but also the portrayal of sister's soft outside while inside strong, not resigned to lonliness's character, a symbol of protagonist's pursuit and yearning for love, dream and life.

The outstanding accordion music in the theme song Catharanthus roseus in the movie Miao Miao directed by the new director Xiaoze Cheng foils the sunshine character of movie heroes, Dai Shi Miao, Xiao Ai incisively and vividly, As if the audience back to the young, confused the but again pure era of high school students with the thoughts movie conveys, find youth and aftertaste moving. In the movie Life of $\mathrm{Pi}$ which repeatedly won the Academy Awards directed by the Chinese director Ang Lee, the opening accordion music--Under Paris Skies foils the youth emotion of Pi the protagonist, in strong contrast to the Pi with emotion changes for many times in the later flowing to the sea so as to highlight the complex inner emotional changes of figures. This movie's winning four awards including best director, Best Visual Effects, Best Musical Score on the 85th Oscar awards ceremony has tight relationship with its diversified music.

Due to the particularity of the theme, the considerations and discussions on factors such as figure construct or plot action in science fiction fantasy movie don't take true feelings of the nature as the rule. However, characters modeling, language expression, action manners etc. in most movies such as movies about literature and life are all close to the natural form of real life, Also only true without artificial performance can more drive the audience's heart. The playing of accordion instrument has beautiful tone quality, wide tone range with abundant expressive force. Through the change of different slow downer, the use of air bellows and the control of finger touch, it can well express all kinds of emotion required in the movie, natural and plain music effect also bring out the best in each 
other with the true feelings shaping of figures the movie want to express. This is the main reason why the accordion playing is constantly brought onto the big screen.

\section{Accordion music and movie national purpose expression}

As one of the main forms of movie art, movie language also occupies a very important position in expressing theme of movie, narrating plot and shaping the characters. Although English is the lingua franca of the world, but in different countries, the language of the movie is basically given priority to their own language. If a movie wanted to go beyond the limitations of national culture and border, the common theme of love, life and death, beliefs is very important. It also requires the theme and story structure and characters are coordinated and real. It has great difficulties. Compared with the movie theme, movie music is a kind of alogical form of sensibility, beautiful music melody is the aesthetic symbol of any country and nation, and more simple. The tone quality of accordion music which is close to nature is full of spiritual communication, easy to cause different national audience's collective sentiments so that reaches the in-depth understanding of artistic charm the movie presents, leaving alone the different national culture, making people between different regional cultures have natural dialogue with the movie.

The director's better understanding of accordion and proper use of accordion in the movie Peacock is also the powerful contribution for the movie to win the German Berlin movie festival awards. In the movie, the accordion music is restraining, fitting in with the time background the movie shows and in agreement with the theme of emotion with mixed feelings of grief and joy of the movie, also according with the intrapersonal aesthetic habits of Chinese people. In addition, either as props of movie plot, or the accordion music, the accordion has a close relationship with the emotion direction of the movie. In the movie, when Zhang Jingchu is imagining her as a paratrooper, accordion plays a kind of cheerful atmosphere and pushes the theme emotion to a climax. When paratrooper falls from the sky, the dream of Zhang Jingchu is disillusioned, the sound of accordion stops abruptly. When Zhang Jingchu's dream of becoming a paratrooper disillusioned, accordion starts to mount once again. The short and strong accordion plays the poor life experience of the figure, making the figure's performance extraordinarily have expressive force and dramatic tension.

The original music One day composed by Ma Shang You in the movie The Beginning of the Great Revival adopts the performance style of accordion. Compared with the previous red revolution background music, the creation of One day is a big progress of Chinese movie music. Guitar playing and accordion music demonstrate a bit smoothness in low and deep, expressing happiness of hope breed in insipid. Unlike the form and style of previous single passionate patriotic songs, the composition of Ma Shang You at this time successfully passes the burden of traditional thinking mode, adopting accordion playing. Simple and quiet movie soundtrack foils well people's love of country and home. At the end of the movie, accordion music also foils the emotion of all being in a calm after great storms our motherland encountered as well as the happiness of people returning to their mother's arms. In movie Railroad Guerrilla, the accordion music recomposing playing of the episode Plunking My Favorite Native Lute makes the performer and listeners be able to understand and grasp the historical background and playing style of the work more accurately.

\section{Conclusion}

Under the era development, the requirement of movie art for television music has also gradually increased, The stand or fall of movie background music has an important decision on the quality of the movie. The changes of accordion music timbre are various, while the sounds great. Under the clever combination of fingers and air bellows, a variety of different styles of music can be played. In the meantime, the accordion music also has diverse forms of performance such as solo, repetition, ensemble etc. This provides more choice space for the creation of movie background music. From the movie art direction, the accordion playing makes their own national culture highly encapsulated into a common thinking paradigm to all mankind with its unique charm of music. It leads the viewers of the 
rest nations in the world to think together and feel the essence of the movie from the depths, showing the nationality and universality of Chinese culture. The accordion music has very high artistic values.

\section{References}

[1] Zhang Xue Mei. The movie “Amelie from Montmartre”music art appreciation, Journal of movie literature, 2014 (18) : 149-150

[2] Liu Mei. Discussion on the symbolic images of accordion music in "Peacock”, Journal of grain in ear, 2014 (14) : 185-186

[3] Yang Guang Dong. Thinking on the development situation of popular accordion in our country in recent years,Journal of Qilu Realm of Arts, 2013 (02) : 29-32

[4] Shi Yibing. The generation of traditional accordion and its historical significance, Journal of Chinese music. 2014 (04) : 218-222

[5] Cai Huifang. The author movie's subjective present--interpretation of the love models and important roles of Wong Kar-wai movie, Journal of movie review, 2006 (17) : 3-6

[6] Wang Huiqin. Discussion on music language communication function of "Life of Pi”, Journal of music creation, 2014 (12) : 143-146

[7] De-jun zhang; Liu Dongxin. Normal universities accordion teaching system reform research, Journal of Qiqihar University (philosophy and social sciences edition); 2011 (03) : 18 to 34 